\title{
Relationship between Physical Activity and Stroke: Review From Different Meta Analytical Studies
}

\author{
Sivachidambaram Kulandaivelan ${ }^{1}$, Sonu Punia ${ }^{1}$ \\ ${ }^{1}$ Assistant Professor, Department of Physiotherapy, GJUS\&T, Hisar.
}

\begin{abstract}
Introduction: Developing countries like India are facing a double burden of communicable and non-communicable diseases. Stroke is one of the leading causes of death and disability in India.

Objective: To systematically review and report the articles from world on physical activity to reduce the risk of stroke.

Methodology: Study was done on May 2016 through various databases using search terms 'physical activity' AND "Meta-analysis" AND 'Stroke'. 17 Meta analytical articles were identified for this review based on inclusion and exclusion criteria.

Results: We selected 17 Meta analysis articles including 137 prospective studies, 541 randomized controlled trials, 22 case control and 108 cohort studies. All articles included in this review were from 2000 to till date. Conclusion: Physical activity is found to have an independent protective effect against cerebrovascular events.
\end{abstract}

\section{Introduction}

Developing countries like India are facing a double burden of communicable and non-communicable disease $^{1,2}$. Stroke is one of the leading causes of death and disability in India. Stroke Morbidity and Mortality in India are as follows: Prevalence 90-222 per 100,000 (Dalal 2007), 102, 620 million deaths (Nongkynrih 2004), 1.44-1.64 million cases of new acute strokes every year (WHO 2005, Murthy 2007), 6,398,000 DALYs (WHO 2009), 12\% of strokes occur in the population aged <40 years (Shah and Mathur 2006) and 28-30 day case fatality ranges from 18-41\% (Dalal 2008, Das 2007). In a recent study conducted in Gujarat, It was found that modifiable risk factors such as hypertension (40\%), alcoholism (35\%), smoking (28\%), hyperlipidemia $(17 \%)$ and physical inactivity are the commonest cause of stroke, and Non-modifiable stroke risk factors include, age, sex, low birth weight, ethnicity and genetic factors.

As a first step the Government of India has started the National Programme for Prevention and Control of Cancer, Diabetes, Cardiovascular Diseases \& Stroke (NPCDCS). The government is focusing on early diagnosis, management, infrastructure, public awareness and capacity building at different levels of health care for all the non-communicable diseases including stroke. An organized effort from both the government and the private sector is needed to tackle the stroke epidemic in India ${ }^{3-8}$.

Regular physical activity has well-established benefits for reducing the risk of premature death and cardiovascular disease. The beneficial effects of physical activity have also been documented for stroke. Fewer studies, however, have investigated the relationship between PA and stroke outcomes, with conflicting and controversial conclusions. Whereas some of these studies reported an inverse association between PA and stroke outcomes, others showed no significant or demonstrated positive associations. So we planned this study to investigate the relationship between physical activity and stroke in meta-analytical studies.

\section{Methodology}

Study was done on May 2016 through various databases using search terms 'physical activity' AND "Meta-analysis" AND 'Stroke'. 17 Meta analytical articles were identified for this review based on inclusion and exclusion criteria.

\section{Results}

We selected 17 Meta analysis articles including 137 prospective studies, 541 randomized controlled trials, 22 case control and 108 cohort studies. All articles included in this review were from 2000 to till date.

Chong Do Lee et al (2004) ${ }^{9}$ had done a Meta-Analysis of Physical Activity and Stroke Risk from 23 studies (18 cohorts and 5 case-controls). Results showed that there was a reduction in stroke risk for active or fit individuals compared with inactive or unfit persons in cohort, case-control, and both study types combined. Highly active individuals had a $21 \%$ lower risk of incident ischemic stroke (RR=0.79; $95 \% \mathrm{CI}, 0.69-0.91)$ and a $34 \%$ lower risk of incident hemorrhagic stroke ( $\mathrm{RR}=0.66$; 95\% CI, 0.48-0.91) compared with low-active individuals. Moderately active individuals also had a $9 \%$ lower risk of incident ischemic stroke (RR $=0.91 ; 95 \%$ CI, 0.80 - 
$1.05)$ and a $15 \%$ lower risk of incident hemorrhagic stroke $(\mathrm{RR}=0.85$; 95\% CI, 0.64-1.13) than did low-active individuals.

Gert Kwakkel et al (2004) ${ }^{\mathbf{1 0}}$ had done a Meta analysis on Effects of Augmented Exercise Therapy Time after Stroke from 32 studies ( 5 studies used a pre-test; post-test assessment design; 3 studies included a control condition but no randomization; and 25 studies were RCTs, with total 2686 stroke patients). A cumulative meta-analysis, adjusted for differences in treatment contrast across all 20 studies, showed a gradual shift from no significant effect in studies with a low treatment contrast ( 0 SDU; CI, -0.30 to $0.30 ; Z=0.0 ; P=1.0)$ to statistically significant overall effect sizes when studies with a high treatment contrast were added (0.13 SDU; 0.03 to $0.23 ; Z=2.49 ; P=0.007)$. Sensitivity analysis revealed significant homogeneous (2_7.07, $P=0.60)$ SES for those studies that applied 15 hours or more $(0.22 \mathrm{SDU} ; 0.07$ to $0.37 ; \mathrm{Z}=2.95 ; P=0.005)$ when compared with studies with a lower treatment contrast $(0.08$ SDU; CI 0.06 to $0.22 ; Z=1.10 ; P=0.136)$. The results of the research synthesis support the hypothesis that augmented exercise therapy has a small but favorable effect on ADL, particularly if therapy input is augmented at least 16 hours within the first 6 months after stroke.

GCW Wendel-Vos et al (2004) ${ }^{\mathbf{1 1}}$ had done a meta-analysis of observational data on Physical activity and stroke from 31 articles (24 cohort and 7 case-control studies). The results for ischaemic stroke were however borderline significant $(P=0.1$; moderately intense physical activity compared with inactivity, showed a protective effect on total stroke for both occupational $(\mathrm{RR}=0.64,95 \% \mathrm{CI}$ : $0.48-0.87)$ and leisure time physical activity $(\mathrm{RR}=0.85,95 \% \mathrm{CI}$ : 0.78-0.93). High level compared with low level leisure time physical activity protected against total stroke $(\mathrm{RR}=0.78,95 \% \mathrm{CI}$ : $0.71-0.85)$, haemorrhagic stroke $(\mathrm{RR}=0.74,95 \%$ CI: $0.57-0.96)$ as well as ischaemic stroke ( $\mathrm{RR}=0.79,95 \% \mathrm{CI}$ : 0.69-0.91). In male populations, being active during leisure time was associated with a 0.54 (95\% CI: 0.36, 0.81) risk of haemorrhagic stroke compared with being inactive during leisure time. In female populations, this risk was 0.76 (95\% CI: 0.67, 0.86). For total stroke, a significant effect $(P=0.008)$ was found for the country under study (either US or Europe). Study concluded that Lack of physical activity is a modifiable risk factor for both total stroke and stroke subtypes. Moderately intense physical activity is sufficient to achieve risk reduction.

Jocelyn E. Harris et al (2010) ${ }^{\mathbf{1 2}}$ had observed that Strength Training Improves Upper-Limb Function in Individuals with Stroke through a Meta- Analysis from 13 articles with 517 individuals. Results showed a positive outcome for strength training was found for grip strength $(\mathrm{SMD}=0.95, P=0.04)$ and upper-limb function $(\mathrm{SMD}=0.21, P=0.03)$. No treatment effect was found for strength training on measures of activities of daily living. A significant effect for strength training on upper-limb function was found for studies including subjects with moderate $(\mathrm{SMD}=0.45, P=0.03)$ and mild $(\mathrm{SMD}=0.26, P=0.01)$ upper-limb motor impairment. There is evidence that strength training can improve upper-limb strength and function without increasing tone or pain in individuals with stroke.

Ming-De Chen et al (2011) ${ }^{\mathbf{1 3}}$ had done a Meta analysis on Effects of Exercise on Quality of Life in Stroke Survivors from 9 studies with 426 stroke survivors. Standardized mean difference (SMD) was used to compute effect size (ES). Exercise can have a small to medium effect on HRQOL outcomes (SMD, 0.32, $P=0.01)$ at post intervention but not at follow-up (12 to 24 weeks post intervention) after exercise was terminated (SMD, 0.17, $P=0.12$ ). Effects of exercise at post intervention showed a significant small to medium ES on overall HRQOL outcomes (grand ES, $0.32 ; 95 \% \mathrm{CI}, 0.12$ to $0.51 ; \mathrm{P}=0.01$ ). Effects at follow-up showed a non significant small ES on HRQOL outcomes (grand ES, 0.17). There was a significant positive ES (grand ES, $0.33 ; P 0.02$ ) on the physical sub domain at post intervention (ie, self-reported changes on strength, bodily pain, and activities of daily living). A similar significant ES (grand ES, 0.23; P 0.04) was found on the mental sub domain at post intervention (ie, self-reported changes on mood and social participation). At follow-up, the effects of exercise declined and there were no significant ESs on both sub domains of HRQOL.

Matthew J Field (2013) ${ }^{\mathbf{1 4}}$ had done a Systematic Review and Meta-analysis on Physical Activity after Stroke from 26 articles $(n=1105)$, of which $11(n=315)$ contained sufficient data for meta-analysis. Steps and Activity Counts Mean number of steps per day ranged from 1,389 $(n=79)$ to 7,379. The random effect summary was 4355.2 steps per day (95\% CI: 3210.4 to 5499.9$)$ with no significant heterogeneity $(Q=10.25, I 2$ $=0$ ). In high-functioning stroke survivors, physical activity including walking was generally low. Strategies are needed to promote and maintain physical activity in stroke survivors. Research is needed to establish reasons for low physical activity after stroke.

Mikhail Saltychev et al (2015) ${ }^{\mathbf{1 5}}$ investigated that aerobic exercises really improve aerobic capacity of stroke survivors by a systematic review and Meta analysis from 13 RCTs (689 participants; 353 cases \&336 
controls). The median size of included study sample was 53(SD 26.5). The synthesis estimates for raw difference between mean difference between changes of means was 2.47 (95\% CI 2.01 to 2.92) $\mathrm{ml} / \mathrm{kg} / \mathrm{min}$. test for sensitivity- the means difference was 1.47 (95\% CI 2.06 to 2.87). Test for exclusion sensitivity- the means difference between 2.22 to $2.58 \mathrm{ml} / \mathrm{kg} / \mathrm{min}$. For subgroup analysis the mean difference between 1.65 and 3.40 $\mathrm{ml} / \mathrm{kg} / \mathrm{min}$. Aerobic training improved aerobic capacity on average by $2.5 \% \mathrm{ml} / \mathrm{kg} / \mathrm{min}$. Strong evidence that aerobic training is capable of improving aerobic capacity of stroke survivors, supporting the routine recommendation for training after stroke.

Marco Y.C. Pang et al (2013) ${ }^{\mathbf{1 6}}$ checked by Using Aerobic Exercise to Improve Health Outcomes and Quality of Life in Stroke from 25 RCT out of which 8 were level 1 study. Meta-analysis showed a significant effect on peak oxygen consumption ( $\mathrm{SMD}=0.55, \mathrm{p}=0.001)$, peak workload $(\mathrm{SMD}=0.77, \mathrm{p}=0.001)$, maximal gait speed $(\mathrm{SMD}=0.37, \mathrm{p}=0.003)$ and walking endurance $(\mathrm{SMD}=0.22, \mathrm{p}=0.001)$ in favor of aerobic exercise. Meta-analysis revealed no significant effect on self-selected gait speed, Berg balance score and Functional Independence Measure score. The efficacy of aerobic exercise in improving other health outcomes in physical, psychosocial and cognitive domains as well as quality of life was inconclusive. There is strong evidence that aerobic exercise (40-50\% HRR progressing to 60-80\%) conducted 20-40 min and 3-5 days per week is beneficial for enhancing aerobic fitness, walking speed and walking endurance in people who have had mild to moderate stroke and are deemed to have low cardiovascular risk with exercise after proper screening assessments (grade A recommendation).

Stoller et al. (2012) ${ }^{\mathbf{1 7}}$ observed effects of cardiovascular exercise early after stroke: systematic review and meta-analysis from 11 trials (10 RCT or randomized controlled pilot studies, 1 study prospective controlled matched design) with 423 participants. Results showed that Peak oxygen uptake $(n=155)$ favoring the intervention group $(\mathrm{SMD}=0.83, \mathrm{CI} 95 \%=0.50-1.16, \mathrm{Z}=4.93, \mathrm{P}<0.01) .6$ Minute Walk Test $(\mathrm{n}=278)$ favoring the cardiovascular training intervention group $(\mathrm{SMD}=0.69, \mathrm{CI} 95 \%=0.45-0.94, \mathrm{Z}=5.58, \mathrm{P}<0.01)$. Gait speed $(\mathrm{n}=243)$ did not show significant results $(\mathrm{SMD}=0.51, \mathrm{CI} 95 \%=-0.25-1.26, \mathrm{Z}=1.31, \mathrm{P}=0.19)$ in favour of early cardiovascular exercise. The syntheses of VO2peak $(\mathrm{I} 2=0 \%, \mathrm{x} 2=0.21, \mathrm{df}=2, \mathrm{P}=0.90)$ and the 6MWT $(\mathrm{I} 2=0 \%, \mathrm{x} 2=2.15, \mathrm{df}=5, \mathrm{P}=0.83)$ results were almost homogenous. They only detected significant heterogeneity for the outcome gait speed $(\mathrm{I} 2=86 \%, \mathrm{x} 2=29.09$, $\mathrm{df}=4, \mathrm{P}<0.01)$. Stroke survivors may benefit from cardiovascular exercise during sub-acute stages to improve peak oxygen uptake and walking distance.

Janne Marieke Veerbeek et al (2014) ${ }^{\mathbf{1 8}}$ had done a Systematic Review and Meta-Analysis from 467 RCTs [n=25373] with 53 interventions. Strong evidence was found for significant positive effects of 13 interventions related to gait, 11 interventions related to arm-hand activities, 1 intervention for ADL, and 3 interventions for physical fitness. Summary Effect Sizes (SESs) ranged from 0.17 (95\%CI 0.03-0.70; I2=0\%) for therapeutic positioning of the paretic arm to $2.47(95 \% \mathrm{CI} 0.84-4.11$; I2 $=77 \%$ ) for training of sitting balance. There is strong evidence that a higher dose of practice is better, with SESs ranging from 0.21 (95\% CI $0.02-0.39 ; \mathrm{I} 2=6 \%)$ for motor function of the paretic arm to $0.61(95 \% \mathrm{CI} 0.41-0.82 ; \mathrm{I} 2=41 \%)$ for muscle strength of the paretic leg.

Carl D. Reimers et al (2009) ${ }^{\mathbf{1 9}}$ had done a review on Exercise as Stroke Prophylaxis from 33 prospective cohort studies and 10 case-control studies. The relative risk (RR) of fatal or non-fatal cerebral infarction is 0.75 , while the corresponding figures for cerebral hemorrhage and stroke of unspecified type are 0.67 and 0.71 , respectively. The reduction of risk is only statistically significant for men. The case-control studies show an RR of 0.32 for men and women combined.

Physical activity is found to have an independent protective effect against cerebrovascular events. The effect is statistically significant only for men, not for women.

James H. Cauraugh et al (2010) ${ }^{20}$ had done a Structured Review and Meta-Analysis on Bilateral Movement Training and Stroke Motor Recovery Progress from 48 stroke studies with 366 stroke patients. A random effects model using the standardized mean difference technique determined a large and significant effect size (0.734; $S E=0.125)$, high fail-safe $N(532)$, and medium variability in the studies $(\mathrm{I} 2=63 \%)$. Moderator variable analysis on the type of bilateral training revealed two large and significant effects: (a) BATRAC (0.842; $S E=0.155)$ and (b) coupled bilateral and EMG-triggered neuromuscular stimulation (1.142; $S E=0.176)$. These novel findings provide strong evidence supporting bilateral arm training with the caveat that two coupled protocols, rhythmic alternating movements and active stimulation, are most effective.

Emma V Cooke et al (2010) $)^{\mathbf{2 1}}$ had done a systematic review and meta-analysis on the effects of increased dose of exercise-based therapies to enhance motor recovery after stroke from 9 papers reporting 7 
studies were included. Single study effect sizes suggest a trend for better recovery with increased dose at the end of therapy but this trend was less evident at follow-up Meta-analysis was possible at outcome for: hand-grip strength, -10.1 [-19.1,-1.2] (2 studies, 97 participants); Action Research Arm Test (ARAT), 0.1 [-5.7,6.0] (3 studies, 126 participants); and comfortable walking speed, 0.3 [0.1,0.5] (2 studies, 58 participants). At followup, between 12 and 26 weeks after start of therapy, meta analysis findings were: Motricity Arm, 10.7 [1.7,19.8] (2 studies, 83 participants); ARAT, 2.2 [-6.0,10.4] (2 studies, 83 participants); Rivermead Mobility, 1.0 [-0.6, 2.5] (2 studies, 83 participants); and comfortable walking speed, 0.2 [0.0,0.4] (2 studies, 60 participants).Current evidence provides some, but limited, support for the hypothesis that a higher dose of the same type of exercisedbased therapy enhances motor recovery after stroke. Prospective dose-finding studies are required.

Lorie G. Richards et al (2008) $)^{22}$ studied 28 articles investigating upper extremity neural representations (e.g., TMS, fMRI, PET, or SPECT) were identified and 13 met inclusion criteria as upper extremity intervention training studies and 166 subjects were tested. The meta-analysis indicated a significant overall mean effect size of 0.84 (S.D. $=0.15$ ) with a $95 \%$ confidence interval ranging from 0.76 to 0.93 . These values indicate that the cumulative effect size is a large effect as well as significant.This large overall effect was based on 13 neural plasticity and motor rehabilitation studies with 98 treatment subjects out of 166 total subjects (i.e., treatment and control groups). Across the studies, the individual effect sizes ranged from a low of -0.54 to a high of 1.95. These results indicate that neural changes in the sensorimotor cortex of the lesioned hemisphere accompany functional paretic upper extremity motor gains achieved with targeted rehabilitation interventions.

Lien Diep, M.D et al (2010) $)^{\mathbf{2 3}}$ had done a Meta-Analysis on Association of Physical Activity Level and Stroke Outcomes in Men and Women from 13 studies. For the total data, the pooled statistical estimate showed an overall significant risk reduction of stroke outcome with moderate to high PA compared with low PA. In particular, compared with low PA, moderate PA resulted in an $11 \%$ reduction in risk of stroke outcome $(\mathrm{RR}=0.89$, CI $0.85-0.94, \mathrm{p}<0.01)$, and high PA resulted in a $21 \%$ reduction in risk $(\mathrm{RR}=0.79$, CI $0.74-0.85$,

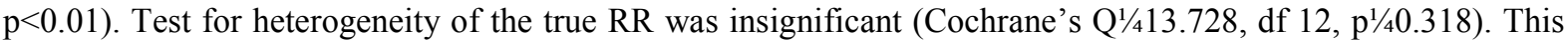
suggests that all studies share a common true effect size. Among the men, the results showed a $12 \%$ reduction in risk of stroke outcome $(\mathrm{RR}=0.88$, CI 0.82-0.94, $\mathrm{p}<0.01)$ for moderate PA and a $19 \%$ reduction for high PA $(\mathrm{RR}=0.81$, CI $0.75-0.87, \mathrm{p}<0.01)$ compared with low PA. Among the women, results showed a $24 \%$ reduction in risk for high PA $(\mathrm{RR}=0.76$, CI 0.64-0.89, $\mathrm{p}<0.01)$ compared with low PA. There was, however, no significant risk reduction associated with a moderate PA level in women.

Jian Li et al (2012) ${ }^{\mathbf{2 4}}$ had done a Meta-Analysis of 21 Prospective Cohort Studies on Physical Activity and Risk of Cardiovascular Disease. Among men, RR of overall CVD in the group with the high level of leisure time PA was 0.76 (95\% CI 0.70-0.82, $p<0.001)$, compared to the reference group with low leisure time PA, with obvious dose-response relationship. A similar effect was observed among women $(\mathrm{RR}=0.73,95 \% \mathrm{CI}$ $0.68-0.78, p<0.001)$. A strong protective effect of occupational PA was observed for moderate level in both men $(\mathrm{RR}=0.89,95 \% \mathrm{CI} 0.82-0.97, p=0.008)$ and women $(\mathrm{RR}=0.83,95 \% \mathrm{CI} 0.67-1.03, p=0.089)$.

M. Lee et al. (2011) ${ }^{\mathbf{2 5}}$ had done a meta-analysis on Presence of baseline prehypertension and risk of incident stroke from 12 Prospective cohort studies with 518,520 participants were included. Prehypertension was associated with risk of stroke (RR $1.55,95 \%$ CI $\left.1.35-1.79 ; p_{-} 0.001\right)$. Among persons with lower-range prehypertension, stroke risk was not significantly increased (RR 1.22, 0.95-1.57). However, for persons with higher values within the prehypertensive range, stroke risk were substantially increased (RR 1.79, 95\% CI 1.492.16). The relative risk from a fixed-effect model was 1.66 (95\% CI $\left.1.57-1.75 ; p \_0.001\right)$.

\section{Discussion}

Meta analytical study concluded that Moderate and high levels of physical activity are associated with reduced risk of total, ischemic, and hemorrhagic strokes. Meta-analytical study's findings suggest that high level of leisure time PA and moderate level of occupational PA have a beneficial effect on cardiovascular health by reducing the overall risk of incident coronary heart disease and stroke among men and women by 20 to 30 percent and 10 to 20 percent, respectively. This evidence from high quality studies supports efforts of primary and secondary prevention of CVD in economically advanced as well as in rapidly developing countries ${ }^{26,27}$.

Stroke is a neglected condition in India. There remains an urgent need for the Indian and state governments to enhance public awareness regarding stoke and preventative measures. A national health promotion policy on for the prevention of risk factors due to stroke would need Evidence-based training, public education of community physicians and health workers in the prevention, diagnosis, management, and rehabilitation of stroke in India so as to reduce the adverse effect on economic development of the country and improve outcome in this region. Early mobilization is considered a priority in order to prevent disabilities and promote function. Further research 
is needed to develop appropriate methods for stroke rehabilitation early after stroke and to evaluate long-term effects of physical activity on aerobic capacity, physical functioning, and quality-of-life.

\section{Conclusion}

Physical activity of mild to moderate intensity is found to have an independent protective effect in general population. Aerobic exercise increase the gait related parameters and endurance, strength training improve upper limb functions in stroke patients.

\section{Reference}

[1]. M. G. Devi, "Epidemiology of neurological disorders in India: Review of background, prevalence and incidence of epilepsy, stroke, Parkinson's disease and tremors," Neurology, Vol 62, Issue 6, pp. 589, 2014.

[2]. J. D. Pandian, A. P. Sudhanb, "Stroke Epidemiology and Stroke Care Services in India” Journal of Stroke, vol. 15, no. 3, pp. 128$134,2013$.

[3]. M. Wasay, I. A. Khatri, S. Kaul, "Stroke in South Asian countries,” Neurol, vol. 10, pp. 135-143, 2014.

[4]. T. K. Banerjee, S. Kumar, "Epidemiology of stroke in India" Neurology Asia, vol.11, pp. 1 - 4, 2011.

[5]. $\quad$ S. K. Das, T. K. Banerjee, "Stroke Indian Scenario," Circulation, vol. 118, pp. 2719-2724, 2008.

[6]. F. C Taylor, S. Kumar, "Stroke Factsheet in India: 2012".

[7]. K Prasad, D. Vibha, Meenakshi, "Cerebrovascular disease in South Asia - Part I: A burning problem," J R Soc Med Cardiovasc Dis, vol. 1, no. 20, 2012

[8]. PM Dalal, "Burden of Stroke - Indian Perspective," JAPI, vol. 52, pp.695, 2004

[9]. C. D. Lee, A. R. Folsom, S. N. Blair, "Physical Activity and Stroke Risk : A Meta-Analysis," Stroke, vol. 34, pp. 2475-2482, 2003.

[10]. G. Kwakkel et al, "Effects of Augmented Exercise Therapy Time after Stroke a Meta-Analysis," Stroke, vol. 35, pp. 2529-2536, 2004.

[11]. G. C. W Wendel-Vos, A. J. Schuit, E. J. M. Feskens, H. C. Boshuizen, W. M. M. Verschuren, W. H. M Saris, D. Kromhout, "Physical activity and stroke. A meta-analysis of observational data," International Journal of Epidemiology, vol. 33, pp. 787-798, 2004

[12]. J. E. Harris et al, "Strength Training Improves Upper-Limb Function in Individuals With Stroke: A Meta-Analysis," 2010.

[13]. M. D. Chen, J. H. Rimmer, "Effects of Exercise on Quality of Life in Stroke Survivors: A Meta-Analysis," Stroke, vol. 42, pp. 832837, 2011.

[14]. M. J Field et al, "Physical Activity after Stroke: A Systematic Review and Meta-analysis,” ISRN Stroke, pp. 13, 2013.

[15]. M. Saltychev et al, "Do aerobic exercises really improve aerobic capacity of stroke survivors? A systematic review and metaanalysis," Euro J Phys Rehabil Med, vol. 2, 2015.

[16]. M. Y. C. Pang, S. A. Charlesworth, R. W.K. Lau, R. C.K. Chung, "Using Aerobic Exercise to Improve Health Outcomes and Quality of Life in Stroke: Evidence-Based Exercise Prescription Recommendations," Cerebrovasc Dis, vol. 35, pp.7-22, 2013.

[17]. Stoller et al, "Effects of cardiovascular exercise early after stroke: systematic review and meta-analysis," BMC Neurology, vol. 12, no. 45,2012

[18]. J.M. Veerbeek, E. V Wegen , R. V. Peppen, P. J. van der Wees , E. Hendriks et al, "What Is the Evidence for Physical Therapy Poststroke? A Systematic Review and Meta-Analysis," PLUS ONE, vol. 9, no. 2, 2014

[19]. C. D. Reimers, G. Knapp, A. K. Reimers, "Exercise as Stroke Prophylaxis,” Deutsches Ärzteblatt International , vol. 106, no. 44, pp. 715-21, 2009

[20]. J. H. Cauraugh, N. Lodha, S. K. Naik, J. J. Summers, "Bilateral Movement Training and Stroke Motor Recovery Progress: A Structured Review and Meta-Analysis," Hum Mov Sci, vol. 29, no. 5, pp. 853-870, 2010.

[21]. E. V. Cooke, K. Mares, A. Clark, R. C Tallis, V. M. Pomeroy, "The effects of increased dose of exercise-based therapies to enhance motor recovery after stroke:a systematic review and meta-analysis," BMC Medicine, vol. 8, pp. 60., 2010.

[22]. L. G. Richards, K. C. Stewart, M. L. Woodbury, C. Senesac, J. H. Cauraugh, "Movement-Dependent Stroke Recovery: A Systematic Review and Meta-Analysis of TMS and fMRI Evidence," Neuropsychologia, vol. 15, issue. 46, no. 1, pp. 3-11, 2008.

[23]. L. Diep, J. Kwagyan, J. Kurantsin-Mills, M, R. Weir, A. J. Trouth, "Association of Physical Activity Level and Stroke Outcomes in Men and Women: A Meta-Analysis,” JOURNAL OF WOMEN'S HEALTH, vol. 19, no. 10, 2010.

[24]. J. Li, J. Siegrist, "Physical Activity and Risk of Cardiovascular Disease-A Meta-Analysis of Prospective Cohort Studies," Int. J. Environ. Res. Public Health, vol. 9, pp. 391-407, 2012

[25]. M. Lee, J. L. Saver, B. Chang, et al, "Presence of baseline prehypertension and risk of incident stroke," Neurology, vol. 77, pp. 1330-1337, 2011

[26]. N. F. Gordon et al, "Physical Activity and Exercise Recommendations for Stroke Survivors: An American", 2004

[27]. L. B. Goldstein et al, "Primary Prevention of Ischemic Stroke. A Guideline from the American Heart Association/American Stroke. Association Stroke Council: Cosponsored by the Atherosclerotic Peripheral Vascular Disease Interdisciplinary Working Group; Cardiovascular Nursing Council; Clinical Cardiology Council; Nutrition, Physical Activity, and Metabolism Council; and the Quality of Care and Outcomes Research. Interdisciplinary Working Group, 2014 